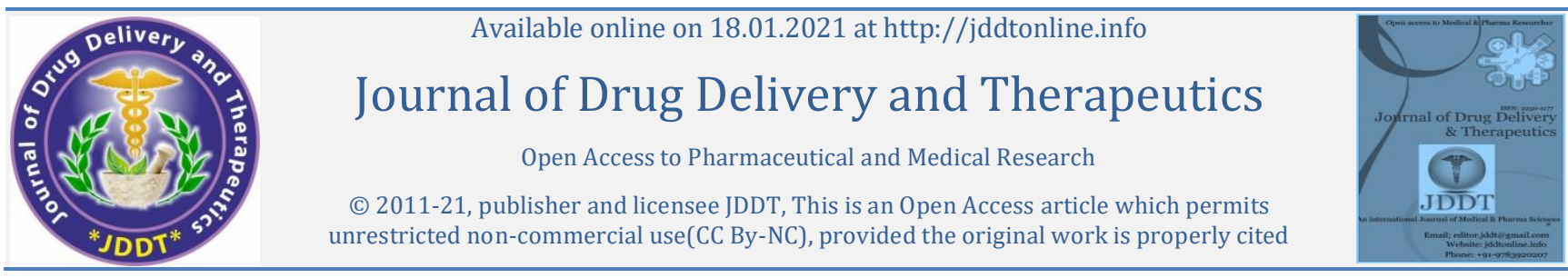

(C) 2011-21, publisher and licensee JDDT, This is an Open Access article which permits Open Access Full Text Article

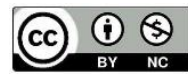

Research Article

\title{
Respiratory function test data and maximum oxygen consumption of children playing football in a hot and humid environment
}

\author{
BAZABA KAYILOU Jean Michel* ${ }^{*}, 2$, AHOUNOU AÏPE Judith ${ }^{1}$, MOUKOUMBI MABIKA Ghislain Aymard 2 \\ ${ }^{1}$ Research Unit Exercise Physiology, Abomey Calavi University. 01BP 918 Cotonou, Benin
}

2 Youth football training school, Gothia- Brazzaville

\begin{tabular}{ll}
\hline Article Info: & $\begin{array}{l}\text { Abstract } \\
\text { Article History: }\end{array}$ \\
Received 23 Oct 2020; \\
Review Completed 26 Dec 2020 \\
Accepted 04 Jan 2021; \\
Available online 18 Jan 2021
\end{tabular}

*Address for Correspondence:

BAZABA KAYILOU Jean Michel, Research Unit Exercise Physiology, Abomey Calavi University. 01BP

Keywords: Training, children, football, spirometry, Congo (Brazzaville)

918 Cotonou, Benin

\section{INTRODUCTION}

Lung volumes and peak expiratory flow rates are related to the morphology of the individual. These measures are widely used in epidemiology and clinical studies. They allow the early detection of certain ventilatory deficits ${ }^{1}$. For this reason, the interpretation of measurements requires knowledge of reference values that depend on gender, height and age. In addition, several factors influence respiratory parameters in children, namely ethnicity, age, environment and level of physical activity. The study by Harik-Khan et $\mathrm{al}^{2}$ showed that respiratory parameters vary according to ethnic and racial groups ${ }^{2}$. Similarly, that of Packa-Tchissambou et $\mathrm{al}^{3}$,showed that the respiratory parameters of Congolese children were lower than those of white children but identical to those in West Africa. Differences are also observed between children of the same ethnicity, sex and age group. The study by Bazaba et $\mathrm{al}^{4}$, showed that female children in rural areas have more developed respiratory parameters than those in urban areas due to certain daily physical survival activities. In addition, puberty is a very important stage in a child's life. It is a dynamic period of development marked by rapid changes in body size, shape and composition ${ }^{5}$. It is characterised, on the one hand, by sexual maturation, which includes the development of secondary sexual characteristics and the acquisition of reproductive function, and, on the other hand, by a growth spurt and morphological changes ${ }^{6}$. The age of onset of morphological changes and the speed of growth vary from child to child, depending on gender, race, ethnicity, environment and nutrition ${ }^{2}$. Similarly, during this period, chest proportions change continuously 7,8 .

In Congo (Brazzaville), a Central African country, the life of the population is characterised by constant impoverishment, the result of a combination of several factors: economic due to the poor distribution of oil revenues and the poor management of the national heritage. To this end, spirometry values would be indispensable for children practising physical activity to explore lung function and to make an orientation on the volume and intensity of training taking into account the usual standards of spirometry values for a given population ${ }^{9,10}$.

In the Republic of Congo, as in most developing countries, football coach-educators have no mastery of training principles due to lack of qualifications. Most of the time, they subject children to physical effort that does not correspond to their stage of development. It is essential to evaluate the respiratory parameters of the Congolese children being trained, based on a local experiment that integrates the various confounding factors mentioned above. The few functional respiratory exploration units operational in Brazzaville are those of the Euro péan respiratory society 1. The lack of Congolese data justified the initiative of this 
preliminary survey, whose main objective is to help determine spirometry equations based on certain physical parameters in the healthy Brazzaville population of children playing football.

\section{MATERIALS AND METHODS}

\section{Topic}

A centre for training children in football was chosen by the non-probabilistic method for this study. The Gothia Brazzaville centre is a socio-educational school that receives children from different backgrounds, socio-economic and ethnic backgrounds. Apart from football, other physical and sports activities are practised, including volleyball, gymnastics, judo, karate and taekwondo. This school has a high number of children thanks to the favourable conditions of access for all. An agreement with ASudh (Action by Relief and Human Development), the Swedish non-governmental organisation (NGO) responsible for the school, allowed us to collect the data.

In order to participate in the study, child footballers should meet the following criteria: be enrolled at the Gothia Brazzaville centre, be regularly attending training sessions over a period of at least 4 months, be between 13 and 17 years old and have parental consent. After an interview with the centre's administration, children who had not reached 4 months of training, those under 13 and over 17 years of age and those presenting a trauma on the day of the test were excluded from the sample. It should be remembered that this centre is located in three districts of the city of Brazzaville with a staff of 1,350 children aged between 8 and 20 years. Ultimately 575 children aged $15 \pm 1.58$ years were selected to participate in the study.

\section{Methods}

A series of pre-experimental matches allowed the subjects to familiarise themselves with the measuring devices during the training sessions. Experimental football matches by age category were then organised for the measurement of respiratory and physical parameters. The test matches were played as official matches in two periods between two teams of the same age group. The duration of the matches and the dimensions of the pitches were fixed by the law of the game at 9 and 11 .

However, in the event of injury to a player selected for the test, substitute players not included in the sample completed the squad to allow for a balance of power between the two teams. The test matches took place in the south of the Republic of Congo (Brazzaville), during the great rainy season at a temperature of $38^{\circ} \mathrm{C}$ and a relative humidity of $60 \%$. On the day of the experiment, two hours before the match, a meal rich in carbohydrates and proteins was provided to the players.
Anthropometric measurements (height, weight) were taken according to the technique recommended by Lohman ${ }^{11}$. Respiratory parameters (FVC, FEV1, FEV1, PEV1) were measured using a portable spirometer of the Autospiro PAL type (Minato, Japan), in seated subjects. Maximum oxygen consumption (V'02max) was obtained using the yoyo IR1 test. All data were collected once by the research team in accordance with the protocols of the American Thoracic Society, ATS ${ }^{8}$. The study was approved by the scientific committee of Marien Ngouabi University (Congo Brazzaville), in accordance with the code of ethics of the International Journal of Sports and Medicine.

\section{Statistical analysis}

Averages are expressed \pm SEM. The statistical processing of the results was carried out using statistical software (SPSS Statistics v 22 IBM corporation, USA).

The Wilcoxon test was used to compare parameters according to age. A two-factor analysis of variance (Friedman) (class vs. measures) was used to compare values as a function of age. When the Friedman analysis was significant, the Wilcoxon test was used for a two-by-two comparison. Pearson correlation analysis was used to determine the relationships between the study variables. The level of significance of the statistical tests was set at $\mathrm{p}<$ 0.05 .

\section{RESULTS}

The values for maximum oxygen consumption ( $\mathrm{V} \mathrm{O} 2 \mathrm{max}$ ) increase considerably with age with extremes of $36.54 \mathrm{l} / \mathrm{min}$, $55.99 \mathrm{l} / \mathrm{min}$. Observation of the values for distance travelled in the yoyoIR1 test according to age shows that children aged 13-14 have low V'02max values, children aged 15-16 have average $\mathrm{V} 02 \mathrm{max}$ values, on the other hand, children aged 17 have good V'02max values.

For the V 02 max as a function of peak expiratory flow there is a disparity of values across age groups, but there is a high representativeness of 15-17 year old children in the class with high PEF values $(\mathrm{n}=240)$.

The classification of $\mathrm{VO} 2 \mathrm{max}$ values according to vital capacity shows a representativeness of 13-14 year old children in the class of low vital capacity values, i.e. 100/140 subjects. However, children aged 15-17 years are representative in the high vital capacity class. As for maximum oxygen consumption as a function of maximum expiratory volume per second, all children aged 13-17 years are represented in the medium FEV1 class (13 years, 50 subjects), ( 14 years, 40 subjects), (15 years, 60 subjects), (16 years, 50 subjects), (17 years, 40 subjects) whereas in the high FEV1 class only children aged 16-17 years are strongly represented, i.e. (16 years, 95 subjects) and (17 years, 80 subjects).

Table 1: Anthropometric characteristics

\begin{tabular}{lllllll}
\hline Age & 13 ans(n=140) & 14 ans(n=120) & 15 ans(n=110) & 16 ans(n=105) & 17 ans(n=100) & p \\
\hline TD & $1,43 \pm 0,06$ & $1,56 \pm 0,06$ & $1,62 \pm 0,08$ & $1,66 \pm 0,07$ & $1,69 \pm 0,07$ & $<0,05$ \\
P & $36,3 \pm 5,95$ & $47,5 \pm 7,7$ & $50,1 \pm 7,55$ & $52,9 \pm 7,23$ & $57,7 \pm 4,96$ & $<0,05$ \\
IMC & $16,06 \pm 1,39$ & $18,87 \pm 2,15$ & $18,81 \pm 1,48$ & $18,93 \pm 1,61$ & $20,21 \pm 1,68$ & $<0,05$ \\
ICO & $52,42 \pm 2,18$ & $50,78 \pm 1,89$ & $51,72 \pm 1,59$ & $51,38 \pm 2,10$ & $50,57 \pm 1,9$ & $<0,05$ \\
AT & $4,30 \pm 1,33$ & $4,20 \pm 1,75$ & $3,66 \pm 1,04$ & $3,40 \pm 1,25$ & $3,10 \pm 1,34$ & $<0,05$
\end{tabular}

$\mathrm{TD}(\mathrm{m})$ : Standing height, P: Weight, BMI (Kg/m2): Body Mass Index, BMI (cm): Body Mass Index, BMI (cm): Cormic Index, BMI $(\mathrm{cm})$ : Chest Magnification 
Table 2: Maximum oxygen consumption by age and vital capacity

\begin{tabular}{lllll}
\hline & CV1 & CV2 & CV3 & $p$ \\
\hline 13 ans & $42,06 \pm 5,87(n=55)$ & $44,81 \pm 0,(n=40$ & $40,34 \pm 1,58(n=45)$ & $<0,05$ \\
14 ans & $38,46 \pm 4,69(n=40)$ & $36,54 \pm 0,00(n=35)$ & $43,11 \pm 6,61(n=45)$ & $<0,05$ \\
15 ans & $46,8 \pm 8,02(n=40)$ & $44,7 \pm 0,79(n=20)$ & $47,94 \pm 5,53(n=50)$ & $<0,05$ \\
16 ans & $48,62 \pm 8,02(n=20)$ & $53,39 \pm 4,86 \quad(n=35)$ & $50,66 \pm 6,18(n=50)$ & $<0,05$ \\
17 ans & - & $55,99 \pm 2,66(n=30)$ & $52,15 \pm 4,54(n=70)$ & $<0,05$ \\
\hline
\end{tabular}

CV1: vital capacity class $1, \mathrm{CV} 2$ : vital capacity class 2 , CV3: vital capacity class 3

Table 3: Maximum oxygen consumption by age and maximum expiratory volume per second

\begin{tabular}{lllll}
\hline & FEV1 & FEV2 & FEV3 & p \\
\hline 13 ans & $42,79 \pm 6,33(\mathrm{n}=70)$ & $41,19 \pm 3,74(\mathrm{n}=70)$ & - & $<0,05$ \\
14 ans & $41,14 \pm 5,31(\mathrm{n}=20)$ & $37,61 \pm 3,63(\mathrm{n}=50)$ & $43,30 \pm 7,61(\mathrm{n}=50)$ & $<0,05$ \\
15 ans & - & $48,53 \pm 6,27(\mathrm{n}=70)$ & $47,80 \pm 6,83(\mathrm{n}=40)$ & $<0,05$ \\
16 ans & - & $50,68 \pm 5,27(\mathrm{n}=45)$ & $50,65 \pm 6,18(\mathrm{n}=60)$ & $<0,05$ \\
17 ans & - & $52,22 \pm 4,83(\mathrm{n}=20)$ & $52,61 \pm 4,58(\mathrm{n}=80)$ & $<0,05$
\end{tabular}

FEV1: class 1 of the maximum expiratory volume per second, FEV2: class 2 of the maximum expiratory volume per second, FEV3: class 3 of the maximum expiratory volume per second.

Table 4: Maximum oxygen consumption by age and peak expiratory flow rate

\begin{tabular}{lllll}
\hline & PEF1 & PEF2 & PEF3 & $\mathrm{p}$ \\
\hline 13 ans & $44,28 \pm 4,98(\mathrm{n}=80)$ & $37,21 \pm 2,84(\mathrm{n}=30)$ & $39,89 \pm 2,21(\mathrm{n}=30)$ & $<0,05$ \\
14 ans & $36,37 \pm 5,31(\mathrm{n}=60)$ & $44,06 \pm 9,14(\mathrm{n}=30)$ & $42,75 \pm 1,61(\mathrm{n}=30)$ & $<0,05$ \\
15 ans & $47,04 \pm 8,52(\mathrm{n}=60)$ & $45,81 \pm 0,78(\mathrm{n}=30)$ & $48,33 \pm 5,83(\mathrm{n}=20)$ & $<0,05$ \\
16 ans & $50,52 \pm 5,22(\mathrm{n}=25)$ & $48,08 \pm 1,03(\mathrm{n}=10)$ & $51,05 \pm 6,40(\mathrm{n}=70)$ & $<0,05$ \\
17 ans & $53,37 \pm 1,04(\mathrm{n}=10)$ & $51,67 \pm 07(\mathrm{n}=10)$ & $52,53 \pm 4,98(\mathrm{n}=80)$ & $<0,05$
\end{tabular}

PEF 1: peak expiratory flow class 1, PEF 2: peak expiratory flow class 2, PEF 3: peak expiratory flow class 3

\section{DISCUSSION}

The present study on the respiratory function of trained Congolese children showed a correlation between anthropometric and respiratory parameters on the one hand, and between respiratory parameters and maximum oxygen consumption during growth on the other. The main results show an increase in maximum consumption according to age and by class as a function of respiratory parameters $(\mathrm{CV}$, FEV1 and PEF).

However, it is important to note some limitations of this work, notably the restriction of the sample of child sportsmen and women to the population of Brazzaville and the failure to take into account the female gender.

Very often, when developing a spirometry equation, the results are compared with reference values established in "normal" or "healthy" subjects. They must have identical anthropometric characteristics to the subject of the study and belong to the same ethnic group ${ }^{12}$. However, most of the equations, using references, were established in European populations with limited age ranges, resulting in significant discontinuities in the transition from one age group to another ${ }^{13}$
Our results concerning maximum oxygen consumption as a function of respiratory parameters in child athletes are in line with those of other authors, particularly for children over 17 years of age 14,15 . This observation is probably related to the intensity of training. It is justified for all respiratory parameters.

We can also assimilate our results to the study by Nourry 16 , who observed exercise-induced hypoxemia in pre-pubertal children. The practice of intensive physical activity in prepubescent children leads to respiratory stress which can have repercussions on the child's respiratory system. Taking into account the physiological principles of training, training at this age should not be based on intensity but rather on play. The child enjoys playing with the ball, which at the same time promotes the harmonious development of physical abilities during growth.

Furthermore, the spirometry equations evaluated in this study are also related to anthropometric and age parameters. This can be explained by several factors such as training, socio-economic and environmental factors ${ }^{17}$. Similarly, certain physical parameters such as V02max also depend on respiratory parameters (CV, FEV1, PEV1), age and the level of sports practice of the subjects. Our results corroborate those of Bacquart et $\mathrm{al}^{18}$, who classified the $\mathrm{V} 02$ max values 
from mediocre to excellent in European trained subjects. A high V 02max in an athlete corresponds to $60 \mathrm{ml} / \mathrm{min} / \mathrm{kg}$, an average V02max for a trained and non-performing athlete depending on the sporting discipline is 40 to $55 \mathrm{ml} / \mathrm{min} / \mathrm{kg}$ and a V $02 \mathrm{max}$ in a sedentary individual is $40 \mathrm{ml} / \mathrm{min} / \mathrm{kg}^{18}$.

In untrained subjects, the diaphragm does not move much and the respiratory rate is 18 to 20 cycles/min. In contrast, in trained subjects, the diaphragm is lowered further and the respiratory rate increases from 6 to 8 cycles/min at rest. The trained subject breathes more economically than untrained subjects for the same working power, he needs less air because he can use a greater proportion of oxygen volume ${ }^{19}$.

Our study carried out on pre-pubescent and pubescent children showed an increase in spirometry and V02max values according to age and level of sports practice. These results corroborate with those obtained by Johnson et al20, on male and female adult subjects practising high level sport.

In this study, we demonstrated that there is a relationship between the spirometry equations and maximum oxygen consumption in trained children. The classification showed that children with a low vital capacity, low peak expiratory volume also had a low V 02 max and those with a medium vital capacity also had a medium V O2max. These results are consistent with the hypothesis that cardiorespiratory values are a function of age, anthropometric values and level of sport participation.

\section{CONCLUSION}

The main objective of this work was to assess the cardiorespiratory profile of young Congolese footballers aged 13-17 years in order to strengthen our understanding of the influence of training on respiratory function. A close relationship was found between the spirometry equations, anthropometric parameters and maximum oxygen consumption in children playing sports. Moreover, these equations are strongly correlated with anthropometric characteristics (Cormic Index, thoracic amplification). Therefore, not all differences observed can be explained by differences in chest dimensions, anthropometric characteristics, the possibility of chest dilatation and lung extensibility.

\section{Conflict of Interest:}

The authors state that they have no conflict of interest in this matter.

\section{CONTRIBUTIONS OF THE AUTHORS}

Bazaba Kayilou JM: corresponding author, project manager. Judith Ahounou CCI: experimental protocol. Ghislain Aymar Moukoumbi Mabika: data collection.

Thanks : Our thanks go to the child footballers and to the NGO Asudh responsible for the youth football training centre (GothiaBrazzaville)

\section{REFERENCES}

1.Quanjer PH, Kubota M, Kobayashi H, Omori H, Tatsumi K, Kanazawa M, et al, Secular changes in relative leg length confound height-based spirometric reference values, Chest, 2015; 147(3):792-7

2. Harik-Khan RI, Muller DC, Wise RA, Racial differences in Lung Function in African-American and white children.Effect of Anthropometric, Socioeconomic, Nutritional and Environmental Factors, Am. J. Epidemiol, 2004; 160:893-900

3. Packa- Tchissambou B, Moulongo AG, Mizere D, Rizet R, Spirometric reference values for Congolese Bantu children and adolescents, Dakar Médical, 2009; 54(2):112-8

4. Bazaba Kayilou JM, Moussouami SI, Massamba A, M’Pemba AB, Mabiala Babela JR, Packa Tchissambou B et al, Spirometric Profile of Congolese Children and Adolescents in Rural and Urban Areas, Int.J.Curr.Res.Aca.Rev, 2016; 4(5):40-50

5. Trabelsi Y, Tabka Z, Richalet JP et al, Spirometric values in Tunisian children. Relationship with pubertal status, Ann. Hum. Biol, 2007; 34:195-205

6. Whitrow MJ, Harding S, Ethnic differences in adolescent lung function: anthropometric, socioeconomic, and psychosocial factors, Am J Respir Crit Care Med, 2008; 177(11):1262-7

7. American Thoraic Society, Standardization of spirometry, Am. J.RespirCrit Care Med, 1995; 152:1107-36

8. Wanger J, Clausen JL, Coates A, Pedersen OF, Brusasco V, Bargos F, Casaburi $\mathrm{R}$ et al, Standarzation of the measurement of lung volumes, Rev Mal Respir, 2007; 24:2S51-64

9. Ben Saad H, Rouatbi S, Raoudha S, Tabka Z, Laouani Kechrid C, Hassen G, et al, Vital capacity and peak expiratory flow rates in a North-African population aged 60 years and over: influence of anthropometric data and parity, Rev Mal Respir, 2003; 20(4):521-30

10. Ben Saad H, El Attar MN, Hadj Mabrouk K, Ben Abdelaziz A, Abdelghani A, Bousarssar $\mathrm{M}$, et al, The recent multi-ethnic global lung initiative (GLI2012) reference values don't reflect contemporary adult's North African spirometry, Respir Med, 2012; 107(12):2000-8

11. Lohman TG, Roche AF, Martorell R, Anthropometrics, Standardization reference manuel: champaign, Human kinestics, 1998; $184 \mathrm{p}$

12. Degens P, Merget R, Reference values for spirometry of the European Coal and Steel Community: time for change, Eur Respir J, 2008; 31(3):687-689

13. Stanojevic S, Wade A, Stocks J, Hankinson J, Coates AL, Pan H et al, Reference ranges for spirometry across all ages: a new approach, Am J Respir Crit Care Med, 2008; 177(3):253-60

14. Massamba A, Litoto PL, Bouyena E, Massamba ES, Moulongo J, Packa TB et al, Données de l'exploration fonctionnelle respiratoire chez des sportifs congolais bantous ages de 20 à 40 ans:étude princeps, Ann. Afr. Med, 2013; 7(1):19-25

15. Vera HM Wanrooij, Maartje Willeboordse, Edward Dompeling,Kim DG Van de Kant. Exercice Training in children with asthma: a systematic review, Br J Sport Med Published. 2012

16. Cédric Nourry, Patrick Mucci, Can pre-pubescent sports children suffer from exercise-induced hypoxemia?, STAPS, 2003; 63 :1928

17. International Child Centre and WHO, Normal Child Development from the Health Team Manual, 1987

18. Bacquart P, Maton F, On your marks, get set, move and be sporty. Ed. Chinon, 2013

19. Bellet M, Grimby G, Barthélémy L. Spirometric studies in French football players, Eur J Appl Physiol, 2006; 98:497-506

20. Johnson BD, Weisman IM, Zeballos RJ, Beck KC, Emerging concepts in the evaluation of ventilatory limitation during exercise: the exrcise tidal flow-volme loop, Chrest, 1999; 116:488-503 